\title{
Minimally invasive biopsy in retroperitoneal tumors (Review)
}

\author{
RADU DRAGOS MARCU ${ }^{1,2}$, CAMELIA CRISTINA DIACONU ${ }^{1,3}$, TRAIAN CONSTANTIN $^{1,4}$, BOGDAN SOCEA ${ }^{1,5}$, \\ FLORENTINA IONITA-RADU ${ }^{6}$, DAN LIVIU DOREL MISCHIANU ${ }^{1,2,7}$ and OVIDIU GABRIEL BRATU ${ }^{1,2,7}$ \\ ${ }^{1}$ University of Medicine and Pharmacy Carol Davila, 050474 Bucharest; \\ ${ }^{2}$ Urology Department, Emergency University Central Military Hospital, 010825 Bucharest; \\ ${ }^{3}$ Internal Medicine Department, Clinical Emergency Hospital of Bucharest, 014461 Bucharest; ${ }^{4}$ Urology Department, \\ 'Prof. Th. Burghele' Clinical Hospital, 050652 Bucharest; ${ }^{5}$ Surgery Department, Pantelimon Emergency Hospital, \\ 021659 Bucharest; ${ }^{6}$ Gastroenterology Department, Emergency University Central Military Hospital, 010825 \\ Bucharest; ${ }^{7}$ Medical Section, Academy of Romanian Scientists, 030167 Bucharest, Romania
}

Received July 17, 2019; Accepted August 9, 2019

DOI: $10.3892 /$ etm.2019.8020

\begin{abstract}
Minimally invasive biopsy procedures have proven over the years to be essential for obtaining a correct diagnosis of retroperitoneal tumors, that allows proper therapeutical conduct. These procedures offer valuable tissue fragments for histopathological examination, that permits the distinction between benign and malignant tumors, identifying the tumors that can benefit from neo-adjuvant treatments, such as chemotherapy or radiotherapy and those that have a direct surgical indication. We have searched the existing data regarding minimally invasive biopsy in retroperitoneal tumors using the PubMed database, in order to evaluate the role of this procedure in establishing a correct diagnosis, as well as to find out the risks of tumor cell seeding and local recurrence after needle biopsy. The risk of tumor cell seeding is very low $(<2 \%)$ and in some cases, it is considered negligible $(<0.5 \%)$. Compared to open biopsy, needle biopsy seems to be associated with a significantly lower risk of tumor cell seeding. According to the existing data, the incidence of needle track tumor cell seeding also depends on the histological type of the tumors. Image-guided retroperitoneal biopsy has proven to be low cost, accessible, and a reliable procedure (in terms of diagnostic accuracy), usually associating with a low rate of complications and a low risk of tumor seeding. Several authors have underlined the importance of the retroperitoneal approach and the association with a co-axial imaging technique in order to avoid potentially deadly complications.
\end{abstract}

Correspondence to: Professor Camelia Cristina Diaconu, University of Medicine and Pharmacy Carol Davila, 8 Eroii Sanitari Street, 050474 Bucharest, Romania

E-mail: drcameliadiaconu@gmail.com

Key words: retroperitoneal tumors, minimally invasive biopsy techniques, tumor cell seeding, tumor recurrence

\section{Contents}

1. Introduction

2. Image-guided tumor biopsy

3. Biopsy techniques

4. Risk of tumor cell seeding using biopsy techniques

5. Conclusions

\section{Introduction}

The retroperitoneal space is often the site of numerous types of tumors, either benign or malignant, that can develop from the retroperitoneal organs, nerves, connective or lymphatic tissues. Many retroperitoneal tumors are asymptomatic, being discovered accidentally during imaging investigations performed for other pathologies, or in advanced stages because of their increased size that may lead to symptoms related to the compression of the nearby organs, with or without their invasion. The histopathological diagnosis has an important role in establishing the correct therapeutical protocol (1). Advanced imaging techniques can provide reliable information regarding the nature of a retroperitoneal mass, favoring therapeutical management without an initial pathological diagnosis. However, these investigations have some limitations (2).

\section{Image-guided tumor biopsy}

The existing data were searched regarding biopsy in minimally invasive retroperitoneal tumors using the PubMed database, in order to evaluate the role of this procedure in establishing a correct diagnosis, and to find the risks of tumor cell seeding and local recurrence after needle biopsy.

\section{Biopsy techniques}

The increasing accessibility of imaging techniques has allowed over the years the development of minimally invasive diagnostic procedures, such as image-guided tumor biopsy. Procedures 
such as needle core biopsy (NCB) or fine needle aspiration biopsy (FNAB), guided using ultrasonography (US) or computed tomography (CT), offer the possibility of obtaining a correct diagnosis, differentiating between benign and malignant tumors and also between metastatic and primary tumors, as well as establishing the tumor subtype. This allows the physician to establish the most appropriate therapeutical management: radiotherapy or chemotherapy, with or without surgery (3).

These procedures are also extremely helpful in advanced tumor stages with unresectable tumors, due to nearby organs invasion or secondary to its metastatic extent, that makes it difficult to achieve a complete oncological excision without any further tumor cell seeding. Ultrasound-guided biopsies, in contrast to those taken under CT guidance, offer the advantage of being more accessible. US-guided biopsies are more accesible, compared to CT-guided biopsies, and avoid radiation exposure. However, factors such as obesity, tumor depth, excessive bowel gas, as well as the type of tissue that must be examined can significantly influence the quality of the images obtained by US $(2,4)$.

The alternative in such cases is represented by CT guidance, that offers a better visualization of the anatomical particularities, with lower risks of image-guided tumor biopsy: pleural, peritoneal and nearby organ perforation, such as liver, spleen, colon, lungs, great vessel (vena cava and aorta injuries with potentially deadly hemorrhages), kidney and urinary tract perforation with secondary retroperitoneal urinoma, which may result in an abscess formation and even urinary sepsis, retroperitoneal abscesses following the accidental perforation of the colon, peritonitis, or pancreatitis (5).

According to the literature, the first CT-guided tumor biopsies date back to the mid-70s, several years after the introduction of CT technology. Shortly thereafter, these procedures became more widely used by physicians, especially after the introduction of multi-slice spiral technology (6). The continuous development of this imaging technology has led to new and more user-friendly CT equipment, with dedicated 3D imaging capture and reconstruction programs, as well as biopsy software and hardware that significantly reduce the time needed for the procedures, thus considerably decreasing the radiation exposure, being increasingly safe and precise. Similar developments have been observed for the US technology, making this imaging technique the first option for image-guided biopsies, due to high accessibility, lack of radiation exposure and the procedure duration. This technique is not flawless, being limited by the thickness of the fatty tissue, as well as by the tumor depth and the presence of intestinal gas. These factors interfere with the ultrasound waves, a phenomenon known as acoustic attenuation, significantly reducing the visibility of the tumor located beneath, as well as of the biopsy needle, which is normally visible, thus increasing the risk of unwanted incidents during biopsy (5).

Biopsy techniques. Regarding tissue sampling procedures, both NCB and FNAB are minimally invasive techniques, that can be used in order to obtain sufficient tissue fragments for the cytopathological examination and ancillary tests such as molecular studies and cytogenetics, polymerase chain reaction (PCR), fluorescent in situ hybridization (FISH), flow cytometry, and microbiological evaluation (6).
FNAB started to be used more frequently in the 1950s, especially in the evaluation process of breast masses, in spite of the fact that it was first reported in the beginning of the 1930s $(7,8)$. The core needle biopsy technique was introduced much later, in the 1990s and it has become more popular due to its several advantages compared to FNAB: it has higher sensitivity and specificity than FNAB, it permits an accurate differentiation between benign and malignant tumors, as well as the distinction between invasive tumors and in situ carcinoma (8). FNAB, image-guided or not (for palpable, superficial tumors), is a fast, low cost, simple and accessible procedure. Usually, it does not require anesthesia, this procedure is associated with little discomfort due to the small gauge needles used, compared to NCB, that uses larger gauge needles. FNAB is less traumatic for tissues than NCB and therefore it has a lower complication rate. In terms of disadvantages, this technique can provide insufficient tissue fragments with poor architectural characteristics, that may prove challenging for molecular testing and immunohistochemistry (6).

Using larger gauge needles and a different tissue sampling technique, NCB provides better tissue fragments, with good architectural characteristics, that are suitable for further molecular and cytogenetic studies, as well as for immunohistochemistry. The quality and the length of the sampled tissue fragments, using NCB, allow the distinction between in situ and invasive tumors, this being not possible in case of FNAB tissue fragments. Therefore, NCB overcomes the disadvantages associated with FNAB. NCB has its own disadvantages: it usually requires image guidance and therefore is not as accessible as FNAB, especially in case of CT guidance, when an interventional radiologist is needed; it also requires a form of anesthesia, because it is a more invasive procedure than FNAB (greater needle gauge, thicker needle, biopsy gun that uses a physical cutting action in order to collect tissue fragments) and it presents a higher risk of bleeding compared to FNAB, as well as infectious complications and pneumothorax (9).

FNAB, followed by immediate on-site tissue examination by a cytopathologist, significantly increases the chances of a correct, quick and complete diagnosis, by indicating the adequacy of the sampled tissue fragments. This technique with immediate on-site examination offers the possibility of obtaining sufficient and proper tissue fragments for further molecular, cytogenetic studies and immunohistochemistry. According to literature, the immediate on-site examination of the tissue fragments obtained using FNAB significantly increases the diagnostic accuracy. Rates of over $95 \%$ have been reported following this technique, as well as decrease of the non-diagnostic biopsies (10).

Another method that can improve the FNAB results is the cell block technique, that consists in processing the tissue fragments obtained with FNAB into paraffin blocks. The block technique significantly improves the diagnostic accuracy, due to the fact that the paraffin block can be cut into numerous sections. Therefore, this technique can provide sufficient material for further examination, which will lead to information on the tissues architectural characteristics and also for ancillary studies, such as immunohistochemistry, cytogenetics, PCR and FISH $(6,11)$.

During FNAB, a negative pressure is applied using a syringe, in order to obtain samples from the suspect lesion. 
This constant negative pressure may traumatize and alter the already sensitive cells, which will lead to further degradation of its architectural characteristics and to diagnostic difficulties. In 1982, Brifford et al introduced the notion of fine-needle non-aspiration cytology, also known as cytopuncture. This procedure is based on the property of capillary tension in a narrow tunnel (11). The technique of cyto-puncture consists in introducing the needle into the lesion, where the mandrel was removed and, afterwards, several movements back and forward were made in order to obtain sufficient material without using a syringe. According to literature, this procedure has proven to be less traumatic for the tissue samples obtained when compared to FNAB. This non-aspiration method provides sufficient material for the examination, with less degraded cells, significantly better architectural characteristics and less blood contamination compared with the aspiration method $(12,13)$.

Even with these described adjuvant techniques, FNAB may prove to be inefficient in terms of obtaining sufficient material for establishing a complete diagnosis, especially when the sampled tissue fragments are too small and insufficient for the cell block technique, thus limiting the cytopathologist's possibility for further ancillary tests. Therefore, the operator's skills and experience are other important factors that can significantly influence the success of this procedure. FNAB cannot differentiate between an in situ neoplasm and an invasive tumor. The reduced amount of tissue obtained with the FNAB technique can hinder the diagnostic process, the incomplete architectural characteristics of the samples often make it difficult for the cytopathologist to differentiate between different types of tumors, such as lymphomas or other lymphoproliferative disorders, like Castleman disease $(14,15)$. In these cases, a NCB may be needed in order to provide sufficient material for an adequate histological diagnosis. In contrast to FNAB, the fragments obtained with the NCB technique preserve the relationship between the lesion and the adjacent normal tissue, therefore allowing the pathologist to assess if the tumor has extended beyond the capsule into the nearby tissue. Other advantages of the NCB approach are that it is superior to FNAB in terms of differentiating between benign lesions, as well as between borderline ones (6).

\section{Risk of tumor cell seeding using biopsy techniques}

An important aspect related to the needle biopsy, that has generated numerous debates and studies, is the risk for needle track tumor seeding. In spite of the fact that this risk is very low $(<2 \%)$ and in some cases considered negligible $(<0.5 \%)$, the risk still exists, and several cases of tumors have been reported that have developed along the needle track after the biopsy was performed $(16,17)$.

A possible explanation for this risk in patients with retroperitoneal tumors may be the fact that in most cases the biopsy is taken using a lumbar/retroperitoneal approach, whereas the tumor excision is made using an anterior approach, either extraperitoneal or intraperitoneal, without removing the biopsy scar during surgery $(18,19)$.

In order to reduce this risk, there are several aspects that should be considered when performing minimally invasive tumor biopsy. The biopsy fragments should be harvested as close as possible to the tumor site, without damaging nearby vital structures, such as the great vessels or adjacent organs, and without contaminating the peritoneal cavity by accidentally puncturing the peritoneum, nor the diaphragm or pleura. Image guidance is essential for a safe and correct procedure, significantly reducing the need for further biopsies, as well as the patient's discomfort, especially when this procedure is performed by an experienced physician (20).

The risk of tumor seeding can be explained by Paget's theory (seed and soil), which stipulates that tumor cell proliferation is stimulated in certain conditions, in the presence of several factors that create a favorable environment. Macrophages, stromal cells and fibroblasts are essential for tumor cell implantation and for the development of a secondary tumor mass. During a needle biopsy, the tumor capsule is perforated and tumor cells may be inseminated alongside the needle track, into the tissues through which the needle passes. Several studies have analyzed the hypothesis that tumor cells can adhere to surgical instruments, as well as to gloves, and that afterward they may be translocated during the surgical procedure to other sites, where due to the presence of favorable conditions they can multiply (21).

Compared to open biopsy, needle biopsy seems to be associated with a significantly lower risk of tumor cell seeding. A 2017 study, concerning the risk of tumor seeding and local recurrence following sarcomas biopsy, has shown a higher incidence of local recurrence in patients who have undergone open biopsy compared to those for whom minimally invasive biopsy was performed (32\% vs. $0.8 \%$ ) (22). In favor of this theory stands the study reported by Sartorelli et al (23) regarding a metastasis developed after thoracoscopic resection of a lung metastasis at the port-site, several months after the procedure was performed. A similar case was reported by Walsh and Nesbitt one year earlier (24). Another interesting case report was published in 2000; the authors presented a case of extremity sarcoma, in which they performed tumor excision with resection of ipsilateral popliteal vessels and vascular reconstruction using the contralateral great saphenous vein. More than one year after surgery, the patient developed metastasis at the site from where the great saphenous vein was harvested, suggesting possible tumor contamination during surgery (25).

According to the existing data, it seems that the incidence of needle track tumor cell seeding also depends on the tumor histological type. It has been reported that hepatocellular tumors account for significantly higher percentages of tumor cell seeding after minimally invasive biopsy compared to other tumors, such as prostatic cancer, breast, thyroid, and pancreatic tumors, the aggressive characteristics of the hepatocellular carcinoma (over $2.5 \%$ compared to less than $1 \%$ ) are well known (21). It seems that the tumor seeding occurs not only after needle biopsy, but also after other minimally invasive procedures (26-28), such as intra-tumoral injections with ethanol and tumor destruction procedures using radiofrequency technique, the mechanism responsible being similar to that involved in needle biopsy (21,29-36).

Wilkinson et al (37) assessed the risk of local recurrence following minimally invasive NCB in patients with retroperitoneal sarcoma. They focused on a subgroup of 150 patients with intermediate and high-grade sarcoma, who had undergone tumor resection. Complete and oncological 
tumor resection was achieved in $85 \%$ of the cases (127 patients), of whom more than half (52\%) developed local recurrence over a median period of time of three years. This group of patients was divided into two smaller groups, in order to establish if there is a difference in terms of tumor local recurrence and overall survival between the cases where NCB was performed before surgery and those without biopsy (90 patients with NCB before surgery vs. 60 patients without NCB). The authors reported that the rate of local recurrence encountered in both groups was similar (52\%), but the median time to recurrence was up to 13 months shorter in the NCB group compared to that encountered in the group without preoperatory NCB (44 months vs. 57 months). No major differences in terms of overall survival were seen between the two groups. Out of the 150 patients who had undergone surgical resection, 96 patients were diagnosed with liposarcoma (using NCB before surgery, and after surgery in the cases without biopsy). The authors did not encounter any differences between the two groups of liposarcoma patients (with and without biopsy) in terms of local recurrence and survival, but they reported significantly lower overall survival rates in the preoperatory biopsy group. The explanation behind these results seems to be the fact that the patients who had undergone NCB presented higher grade tumors compared to those without biopsy, who presented lower grade tumors, with imaging characteristics that made them easier to recognize and therefore a preoperatory biopsy was not necessary. Thus, the difference in terms of overall survival in the liposarcoma group is related to the tumor grade and not to the NCB (37).

Another smaller study, which evaluated the impact of NCB in terms of safety and risk of local recurrence in patients with retroperitoneal sarcoma, reached similar outcomes. The authors analyzed 22 cases in which NCB were performed before surgery. The procedure was done under CT guidance, using a transperitoneal approach. The diagnosis was accurate in 18 patients $(82 \%)$, in two cases the tissue fragments were inconclusive for the diagnosis and in further two cases the diagnosis of schwannoma and gastrointestinal stromal tumor was established, that later proved to be sarcoma. The sarcoma subtype was identified in only 6 patients, whereas the tumor grade was reported in half of the cases. The two most frequent sarcoma subtypes identified using NCB were liposarcoma and pleomorphic sarcoma, each accounting for $27 \%$, followed by leiomyosarcoma (18\%) and other subtypes such as synovial sarcoma, spindle cell sarcoma, solitary fibrous tumor, each representing less than 9\%. During the follow-up period, the authors did not encounter any complications, nor local tumor recurrence or signs of tumor seeding after biopsy (38).

A much larger prospective study was published in 2017 , conducted on 498 patients with retroperitoneal sarcoma who had undergone surgical resection. In more than $50 \%$ of the cases (255 patients), a preoperatory NCB was performed in order to obtain a diagnosis. During the follow-up examinations, it was found that $2 \%$ of the patients included in the NCB group (5 patients) developed tumor recurrence along the needle track and that two patients also presented distant mestastasis. In these recurrence cases, a transabdominal approach was used for the biopsy, but the authors reported that there were no differences in terms of risk for local recurrence between the retroperitoneal approach and the transabdominal pathway. An important factor that seemed to be associated with the risk of recurrence was the fact that the procedure was performed without a co-axial technique. The authors mentioned that the patients with retroperitoneal liposarcoma were less likely to undergo biopsy, due to the imaging characteristics of the liposarcoma that made it easier to recognize. Therefore, the predominant sarcoma subtype in the non-NCB group was liposarcoma, accounting for $87.7 \%$ of cases, whereas in the NCB group liposarcoma was found in $44.5 \%$ patients. The second most frequent tumor subtype was leiomyosarcoma (representing $17.9 \%$ of the 498 patients), being more frequently encountered in the NCB group. Other sarcoma subtypes, such as pleomorphic sarcoma, synovial sarcoma, solitary fibrous tumor, each accounted for $<5 \%$ (20).

\section{Conclusions}

Minimally invasive tumor biopsy is often a necessary procedure, that could guide towards the diagnosis, especially in cases of retroperitoneal masses without typical imaging characteristics. According to literature, the risk of tumor seeding and local recurrence after a minimally invasive biopsy is low, but not negligible, several cases of tumor recurrence after NCB have been reported. Several studies have underlined the fact that the preferred route when considering a retroperitoneal tumor biopsy should be the retroperitoneal approach, due to the fact that it reduces the risks of biopsy incidents, such as intraperitoneal organ puncture, as well as the risk of intraperitoneal tumor seeding. Image guidance, either US or CT examinations, is essential for a safe and correct procedure, as well as for guiding the needle towards the tumor, in order to obtain sufficient fragments for diagnosis, therefore significantly reducing the need for further biopsy and patient discomfort. It has been reported that CT co-axial techniques are associated with lower risk of biopsy failure and also fewer complications, such as needle tract tumor recurrence.

\section{Acknowledgements}

Not applicable.

Funding

No funding was received.

\section{Availability of data and materials}

Not applicable.

\section{Authors' contributions}

RDM, TC, BS and OGB collected, analyzed and interpreted the data regarding the minimally invasive biopsy in retroperitoneal tumors. CCD, DLDM and FIR had substantial contribution to the conception of the study and interpretation of data; also, they drafted the manuscript and were major contributors in writing the manuscript. All authors read and approved the final manuscript. 


\section{Ethics approval and consent to participate}

Not applicable.

\section{Patient consent for publication}

Not applicable.

\section{Competing interests}

The authors declare that they have no competing interests.

\section{References}

1. Van Roggen JFG and Hogendoorn PC: Soft tissue tumours of the retroperitoneum. Sarcoma 4: 17-26, 2000.

2. Mehdi G, Maheshwari V, Afzal S, Ansari HA and Ahmad I: Image-guided fine-needle aspiration of retroperitoneal masses: The role of the cytopathologist. J Cytol 30: 36-41, 2013.

3. Das C, Sengupta M, Mukhopadhyay M and Saha AK: Critical clinical appraisal of the role of computed tomography-guided minimally invasive aspiration cytology in evaluation of retroperitoneal masses. Indian J Med Paediatr Oncol 35: 60-65, 2014

4. Guo Z, Kurtycz DF, De Las Casas LE and Hoerl HD Radiologically guided percutaneous fine-needle aspiration biopsy of pelvic and retroperitoneal masses: A retrospective study of 68 cases. Diagn Cytopathol 25: 43-49, 2001.

5. Micames C, Jowell PS, White R, Paulson E, Nelson R, Morse M, Hurwitz H, Pappas T, Tyler D and McGrath K: Lower frequency of peritoneal carcinomatosis in patients with pancreatic cancer diagnosed by EUS-guided FNA vs. percutaneous FNA Gastrointest Endosc 58: 690-695, 2003.

6. Paquin SC, Gariépy G, Lepanto L, Bourdages R, Raymond G and Sahai AV: A first report of tumor seeding because of EUS-guided FNA of a pancreatic adenocarcinoma. Gastrointest Endosc 61: 610-611, 2005

7. Roman A, Achimas-Cadariu P, Fetica B, Gata V and Seicean A CT-guided procedures: An initial experience. Clujul Med 91: 427-434, 2018.

8. Golse N, Ducerf C, Rode A, Gouillat C, Baulieux J and Mabrut JY: Transthoracic approach for liver tumors. J Visc Surg 149: e11-e22, 2012.

9. Joudeh AA, Shareef SQ and Al-Abbadi MA: Fine-needle aspiration followed by core-needle biopsy in the same setting: Modifying our approach. Acta Cytol 60: 1-13, 2016.

10. Misra RK, Mitra S, Jain RK, Vahikar S, Bundela A and Misra P. Image-guided fine needle cytology with aspiration versus non-aspiration in retroperitoneal masses: Is aspiration necessary? J Pathol Transl Med 49: 129-135, 2015.

11. Briffod M, Gentile A and Hébert H: Cytopuncture in the follow-up of breast carcinoma. Acta Cytol 26: 195-200, 1982

12. VanderLaan PA: Fine-needle aspiration and core needle biopsy: An update on 2 common minimally invasive tissue sampling modalities. Cancer Cytopathol 124: 862-870, 2016.

13. Nasuti JF, Gupta PK and Baloch ZW: Diagnostic value and cost-effectiveness of on-site evaluation of fine-needle aspiration specimens: Review of 5,688 cases. Diagn Cytopathol 27: 1-4, 2002.

14. Krogerus L and Kholová I: Cell block in cytological diagnostics: Review of preparatory techniques. Acta Cytol 62: 237-243, 2018

15. Łukasiewicz E, Ziemiecka A, Jakubowski W, Vojinovic J, Bogucevska M and Dobruch-Sobczak K: Fine-needle versus core-needle biopsy - which one to choose in preoperative assessment of focal lesions in the breasts? Literature review. J Ultrason 17: 267-274, 2017.

16. Raghuveer CV, Leekha I, Pai MR and Adhikari P: Fine needle aspiration cytology versus fine needle sampling without aspiration. A prospective study of 200 cases. Indian J Med Sci 56: 431-439, 2002.

17. Pinki P, Alok D, Ranjan A and Nanak Chand M: Fine needle aspiration cytology versus fine needle capillary sampling in cytological diagnosis of thyroid lesion. Iran J Pathol 10: 47-53, 2015.
18. Monaco SE, Khalbuss WE and Pantanowitz L: Benign non-infectious causes of lymphadenopathy: A review of cytomorphology and differential diagnosis. Diagn Cytopathol 40: 925-938, 2012

19. Meyer L, Gibbons D, Ashfaq R, Vuitch F and Saboorian MH Fine-needle aspiration findings in Castleman's disease. Diagn Cytopathol 21: 57-60, 1999.

20. Van Houdt WJ, Schrijver AM, Cohen-Hallaleh RB, Memos N, Fotiadis N, Smith MJ, Hayes AJ, Van Coevorden F and Strauss DC: Needle tract seeding following core biopsies in retroperitoneal sarcoma. Eur J Surg Oncol 43: 1740-1745, 2017.

21. Berger-Richardson D and Swallow CJ: Needle tract seeding after percutaneous biopsy of sarcoma: Risk/benefit considerations. Cancer 123: 560-567, 2017.

22. Barrientos-Ruiz I, Ortiz-Cruz EJ, Serrano-Montilla J, Bernabeu-Taboada D and Pozo-Kreilinger JJ: Are biopsy tracts a concern for seeding and local recurrence in sarcomas? Clin Orthop Relat Res 475: 511-518, 2017.

23. Sartorelli KH, Partrick D and Meagher DP Jr: Port-site recurrence after thoracoscopic resection of pulmonary metastasis owing to osteogenic sarcoma. J Pediatr Surg 31: 1443-1444, 1996.

24. Walsh GL and Nesbitt JC: Tumor implants after thoracoscopic resection of a metastatic sarcoma. Ann Thorac Surg 59: 215-216, 1995.

25. Hughes TM and Thomas JM: Sarcoma metastases due to iatrogenic implantation. Eur J Surg Oncol 26: 50-52, 2000.

26. Socea LI, Visan DC, Barbuceanu SF, Apostol TV, Bratu OG and Socea B: The antioxidant activity of some acylhydrazones with dibenzo[a,d][7]annulene moiety. Rev Chim (Bucharest) 69: 795-797, 2018.

27. Radulescu A, Madan V, Aungurenci A, Bratu O, Farcas C, Dinu $\mathrm{M}$ and Mischianu D: Antibiotic resistant urinary tract infections in an urology ward. Rom J Mil Med 118: 20-22, 2015.

28. Spinu D, Bratu O, Popescu R, Marcu D, Radulescu A and Mischianu D: Clostridium difficile - an emerging plague. Rom J Mil Med 118: 12-15, 2015.

29. Lupu M, Caruntu A, Caruntu C, Papagheorghe LML, Ilie MA, Voiculescu V, Boda D, Constantin C, Tanase C, Sifaki M, et al: Neuroendocrine factors: The missing link in non melanoma skin cancer (Review). Oncol Rep 38: 1327-1340, 2017.

30. Neagu M, Caruntu C, Constantin C, Boda D, Zurac S, Spandidos DA and Tsatsakis AM: Chemically induced skin carcinogenesis: Updates in experimental models (Review). Oncol Rep 35: 2516-2528, 2016.

31. Boda D: Cellomics as integrative omics for cancer. Curr Proteomics 10: 237-245, 2013.

32. Neagu M, Constantin C, Tanase C and Boda D: Patented biomarker panels in early detection of cancer. Recent Pat Biomark 1: 10-24, 2011

33. Boda D, Docea AO, Calina D, Ilie MA, Caruntu C, Zurac S, Neagu M, Constantin C, Branisteanu DE, Voiculescu V, et al: Human papilloma virus: Apprehending the link with carcinogenesis and unveiling new research avenues (Review). Int J Oncol 52: 637-655, 2018.

34. Solomon I, Voiculescu VM, Caruntu C, Lupu M, Popa A, Ilie MA, Albulescu R, Caruntu A, Tanase C, Constantin C, et al: Neuroendocrine factors and head and neck squamous cell carcinoma: An affair to remember. Dis Markers 2018: 9787831 , 2018.

35. Lupu M, Caruntu A, Caruntu C, Boda D, Moraru L, Voiculescu V and Bastian A: Non-invasive imaging of actinic cheilitis and squamous cell carcinoma of the lip. Mol Clin Oncol 8: 640-646, 2018.

36. Voiculescu VM, Caruntu C, Solomon I, Lupu M, Ilie MA, Boda D, Constantin C and Neagu M: Squamous cell carcinoma: Biomarkers and potential therapeutic targets. In: Human Skin Cancers-Pathways, Mechanisms, Targets and Treatments. Blumenberg M (ed). IntechOpen, London: pp135-159, 2018.

37. Wilkinson MJ, Martin JL, Khan AA, Hayes AJ, Thomas JM and Strauss DC: Percutaneous core needle biopsy in retroperitoneal sarcomas does not influence local recurrence or overall survival. Ann Surg Oncol 22: 853-858, 2015.

38. Hwang SY, Warrier S, Thompson S, Davidson T, Yang JL and Crowe P: Safety and accuracy of core biopsy in retroperitoneal sarcomas. Asia Pac J Clin Oncol 12: e174-e178, 2016. 